\section{$\underset{\substack{\text { hommes } \\ \text { \& migrations }}}{ }$}

\section{Hommes \& migrations}

Revue française de référence sur les dynamiques

migratoires

$1304 \mid 2013$

Frontières

\title{
Calais, une prison ouverte pour les migrants
}

\section{Brice Arsène Mankou}

\section{CpenEdition \\ Journals}

\section{Édition électronique}

URL : http://journals.openedition.org/hommesmigrations/2634

DOI : 10.4000/hommesmigrations.2634

ISSN : 2262-3353

\section{Éditeur}

Musée national de l'histoire de l'immigration

\section{Édition imprimée}

Date de publication : 1 octobre 2013

Pagination : 35-39

ISBN : 978-2-919040-24-7

ISSN : $1142-852 X$

\section{Référence électronique}

Brice Arsène Mankou, "Calais, une prison ouverte pour les migrants », Hommes \& migrations [En ligne], 1304 | 2013, mis en ligne le 01 janvier 2017, consulté le 10 décembre 2020. URL : http://

journals.openedition.org/hommesmigrations/2634; DOI : https://doi.org/10.4000/

hommesmigrations.2634 
Jeune afghan tentant de s'introduire dans un poids

lourd sur l'autoroute qui mène au terminal des ferries

du port de Calais. Les jours de tempête en mer, le trafic

sur l'autoroute est tellement dense que les camions

sont quasiment à l'arrêt, Calais, France.

๑) Mathias Depardon 


\title{
CALAIS, \\ UNE PRISON OUVERTE POUR LES MIGRANTS
}

par BRICE ARSÈNE MANKOU, docteur en sociologie, université de Lille-I, enseignant à l'université du Littoral-Côte d'Opale de Dunkerque, chercheur associé au Clersé, CNRS (UMR 8019).

\author{
Depuis la fermeture du camp de Sangatte en 2003 et \\ la destruction de la jungle de Calais qui a suivi la situation \\ des migrants à la frontière franco-britannique est devenue \\ encore plus complexe et violente. Condamnés à une attente \\ sans but, criminalisés, ne devant leur difficile subsistance \\ qu'à l'aide des bénévoles, ces migrants expérimentent \\ de nouvelles modalités de l'enfermement. Voici qu'éclate \\ le paradoxe contemporain de la frontière, à la fois zone \\ de passage impossible et no man's land sans issue.
}

\section{Des migrants enfermés à l'extérieur}

Le quotidien des migrants afghans, irakiens, iraniens, érythréens à Calais est insupportable. Ils ne sont ni les bienvenus au Royaume-Uni, ni en France. Ils ne sont ni régularisables ni expulsables. C'est ce que Marc Bernardot décrit comme "les nouvelles frontières" où l'État déclare "la guerre aux migrants" par un système de surveillance.

Notre étude, qui se veut empirique, s'appuie sur une enquête que nous avons menée en 2012 auprès des bénévoles du Secours catholique, de Salam, de la Croix-Rouge et de l'association CSUR qui viennent en aide à ces migrants. Dans une perspective sociologique, nous voulons analyser cette frontière "ouverte et fermée" pour des migrants "enfer- més dehors". Un accent particulier sera mis sur cette forme de frontière ou de prison et sur le travail des responsables associatifs qui contribuent à les aider à Calais. Nous mettrons aussi en évidence ces entretiens réalisés sur cette frontière particulière qui n'entame pas leur détermination à vouloir la franchir. Pour ces migrants, souligne Yvetta Sartana: "Nous n'avons pas violé la frontière, c'est la frontière qui nous a violés.".

L'érection de l'Europe en forteresse constitue une remise en cause des accords de Schengen. C'est désormais l'épouvantail de la France "forteresse" qui est brandi pour susciter des peurs à tort ou à raison. Dans ce contexte, les migrants de Calais, 
victimes de la fermeture de Sangatte, errent dans les rues de cette ville, où les associations tentent de leur venir en aide. Lépisode de la fermeture de Sangatte est encore présent dans les mémoires. Aujourd'hui, ces migrants connaissent un type d'exil forcé, car ils ne peuvent ni rester en France ni entrer au Royaume-Uni. Est-ce que ces migrants sont dans une prison ouverte à Calais ? C'est à cette principale question que nous répondrons.

La problématique de l'errance ou de l'exil ${ }^{4}$ forcé des migrants de Calais est une préoccupation lancinante des pouvoirs publics. En effet, cette situation pose clairement les questions liées à la circulation migratoire à l'intérieur des frontières européennes et celles des modalités de l'enfermement qui jouent dans la circulation de ces migrants tantôt "enfermés dehors", tantôt "enfermés dedans". Cette dialectique du "dedans" et du "dehors" n'a qu'un dominateur commun, la privation de la liberté d'aller et de venir. C'est ce que Marc Bernardot appelle "linstitutionnalisation de la guerre aux migrants et aux minorités"

Pour mener cette lutte contre l'immigration clandestine, le choix de la frontière comme lieu de la guerre déclarée aux migrants n'est ni anodin ni neutre. Très présente en géographie, la notion de frontière est entrée tout récemment dans les sciences sociales, à partir du moment où la mondialisation a renforcé les mobilités du Sud vers le Nord. La sociologie des migrations, depuis les théories de l'École de Chicago, a permis d'inscrire les frontières comme une catégorie essentielle de la recherche.

À partir de létude sur les migrants de Calais, nous poursuivons deux principaux objectifs : démontrer les liens entre la notion de frontière et celle de migration, étant entendu qu'il ne saurait y avoir de mobilité sans qu'elle passe par les frontières ; analyser les conséquences du confinement des migrants du fait de l'apparition de nouvelles frontières de la mondialisation.

Les cas de Sangatte hier et de Calais aujourd'hui illustrent bien ces conséquences qui se manifestent par l'enfermement, l'assignation et les différentes formes de privation de libertés dont celle d'aller et de venir sur le territoire français. Notre enquête a permis de mieux connaître ces migrants, notamment leur âge, leur pays d'origine et les raisons pour lesquelles ils sont partis de leur pays.

\section{Calais, une frontière de la mondialisation en question}

Affirmer que Calais est une nouvelle frontière de la mondialisation revient à circonscrire le contexte dans lequel cette frontière s'est construite. En effet, c'est à partir de l'effondrement du mur de Berlin et de la reconfiguration des équilibres géostratégiques entre les États-Unis et l'ex-bloc de l'URSS que les questions de circulation des peuples fuyant des zones en conflit ont émergé. Avant Calais, Sangatte avait pour objectif principal le maintien des migrants sur le territoire du département du Pas-de-Calais, des accords entre la France et le Royaume-Uni empêchant ces migrants de passer outre-Manche.

Aujourd'hui, la réalité des frontières permet non seulement aux États de maintenir des migrants sur leur territoire, mais aussi de coopérer avec d'autres pour expulser des migrants dans leur pays d'origine ou encore dans un pays tiers. L'uniformisation des directives européennes en matière d'immigration permet désormais un meilleur contrôle des frontières entre États. C'est le cas des demandeurs d'asile dans les pays de l'espace Schengen, par exemple, avec l'organisation de la traçabilité de leur première demande d'asile.

Calais est par conséquent une frontière de la mondialisation où les migrants peuvent être enfermés, assignés et confinés. Les frontières de la mondialisation rendent problématique le droit à la mobilité qui ne semble pas faire l'unanimité, y compris dans 
les pays européens comme la France, vu la situation critique des migrants de Calais depuis la fermeture du camp de Sangatte en 2003.

La ville-frontière de Calais, en dépit de la destruction de la jungle en 2009, continue de faire face à la présence des migrants. Visibles dans les villes voisines, les migrants de Calais ne veulent pas rester en France mais entrer au Royaume-Uni. Avec la fermeture de Sangatte, les squatts dans des hangars et autres refuges de fortune leur permettent de passer des journées entières à l'abri.

\section{Un lieu mythique de la guerre et des migrants enfermés "dehors"}

Plusieurs travaux éclairants se sont intéressés aux processus de confinement et d'enfermement des migrants dans l'espace européen. Ceux de Carolina Kobelinsky et de Chowra Makaremi ${ }^{6}$, notamment, décrivent les mécanismes du confinement et de l'enfermement des migrants. Pour Chowra Makaremi, qui s'inscrit dans la perspective des travaux de Michel Foucault sur le biopouvoir, l'usage de la force et de la violence traduit bien cette guerre déclarée aux migrants. Quant à Carolina Kobelinsky, sa description de l'expérience d'un demandeur d'asile montre la complexité tout autant de la circulation que de l'enfermement des migrants dans les frontières.

À Calais, la guerre aux migrants se matérialise par la brutalité avec laquelle ont eu lieu en 2003 la fermeture de Sangatte par le ministre de l'Intérieur d'alors, Nicolas Sarkozy, et celle de la "jungle" le 22 avril 2009 par le ministre de l'Immigration, Éric Besson. C'est ce que Chowra Makaremi appelle "une pénalisation de la circulation et des reconfigurations de la frontière" par des migrants kurdes, afghans, érythréens, soudanais, éthiopiens et somaliens, qui veulent se rendre au Royaume-Uni et qui, malgré eux, sont maintenus et assignés à Calais.
Cette assignation fait de Calais une prison ouverte pour ces hommes et ces femmes confinés à sa frontière. Pourtant, le règlement de Dublin n ${ }^{\circ}$ 343/2003 du Conseil du 18 février 2003 souligne que, "à la demande d'un État membre, tout État membre peut accepter d'examiner une demande d'asile dont il n'est pas responsable et ce pour des raisons humanitaires fondées notamment sur des motifs familiaux ou culturels, à condition que les intéressés le souhaitent...". Cette disposition n'est pas souvent rappelée aux migrants qui restent sans droit en France.

Ce sont ces paradoxes que dénonce Michel Agier, lorsqu'il souligne que "le monde contemporain, celui que nous vivons et qu'il nous est difficile de penser, est soumis à un paradoxe intellectuel. Les mots les plus souvent convoqués pour l'identifier sont ceux de globalisation et de la violence des États sur les gens, par des frontières souvent faites de murs et par la violence d'identités collectives souvent dirigées contre l'autre ${ }^{7 "}$. La frontière de Calais n'est plus cet espace géographique qui sépare la France du Royaume-Uni, mais le symbole des contrôles d'identité, des interpellations et des conduites au commissariat, notamment à Coquelles, siège de la police des airs et des frontières, faisant de ces migrants des personae non gratae en terre française, où la population assimile souvent leur errance à une simple question de clandestinité ou de sans-papiers. On se souvient encore des manifestations de juin 2009 : des militants hostiles à la politique européenne d'immigration avec le slogan "No border", c'est-à-dire "contre les frontières", réclamaient la liberté de circulation contre des politiques migratoires humiliantes, inhumaines et dégradantes.

La privation de libertés des migrants à la frontière de Calais signifie qu'ils ne se trouvent ni en France ni au Royaume-Uni. Comme le souligne Stéphane Julinet: "Jusqu'en 1992, l'État français estimait que les étrangers non admis n'étaient pas entrés en France: ils se trouvaient dans une zone internationale où la loi française ne s'appliquait pas et où ils pouvaient donc être maintenus sans règle, sans limite et 
sans contrôle $e^{9}$. La guerre contre les migrants passe par leur enfermement, leur confinement dans une prison ouverte.

\section{Situation des migrants de Calais : une enquête sur le terrain}

La plupart des migrants que nous avons rencontrés à Calais, grâce au partenariat avec les associations humanitaires, sont originaires d'Afghanistan, du Kurdistan, de l'Irak, d'Iran ${ }^{10}$, de la Corne de l'Afrique et du Darfour. Grâce aux bénévoles d'associations, nous avons établi des liens avec les migrants et nous nous sommes entretenus avec certains d'entre eux qui comprennent plus ou moins le français. Nous avons réalisé des entretiens non enregistrés, en mars 2012, avec 50 migrants. Ces entretiens semi-directifs duraient généralement une demi-heure et se déroulaient dans les locaux du Secours catholique. Ils ont permis de cerner leur âge, leur pays d'origine et les raisons qui les ont poussés à quitter leurs pays. Nous n'avions pas de questionnaire d'entretien, l'objectif pour nous étant d'observer et de comprendre les trajectoires de ces migrants de Calais.

Près de la moitié d'entre eux ont entre 25 et 35 ans. La plupart sont des hommes qui ont un niveau d'études primaires voire secondaires. Très peu ont un niveau universitaire.

\section{Tableau 1 : Répartition par âge des migrants interviewés.}

\begin{tabular}{|c|c|}
\hline Tranche d'âge & Nombre d'enquêtés \\
\hline $15-20$ ans & 5 \\
\hline $21-25$ ans & 10 \\
\hline $25-35$ ans & 30 \\
\hline $35-40$ ans & 5 \\
\hline Total & $\mathbf{5 0}$ \\
\hline
\end{tabular}

Il n'est pas surprenant que la plupart de nos enquêtés soient originaires des pays déstabilisés par les guerres, comme l'Afghanistan, la Tchétchénie, l'Irak, le Soudan et la Somalie. À noter également, l'implication des passeurs qui exploitent la misère de ces migrants.

\section{Tableau 2 : Répartition des pays d'origine des migrants.}

\begin{tabular}{|c|c|}
\hline Pays d'origine & Nombre d'enquêtés \\
\hline Afghanistan & 30 \\
\hline Tchétchènie & 5 \\
\hline Kurdistan & 5 \\
\hline Irak & 3 \\
\hline Iran & 2 \\
\hline Soudan & 3 \\
\hline Somalie & 2 \\
\hline Total & $\mathbf{5 0}$ \\
\hline
\end{tabular}

Parmi les facteurs qui ont poussé ces migrants à s'enfuir de leur pays, figurent en bonne place la guerre, l'enrôlement dans les milices, les persécutions politiques et la misère. Si, pour la plupart des migrants afghans, tchétchènes, irakiens, la guerre est le facteur déterminant de leur migration, dans les pays du Darfour comme le Soudan, en revanche, l'enrôlement dans les milices armées et les persécutions politiques en sont les principales causes.

\begin{tabular}{|c|c|}
\hline Facteurs de migration & Nombre d'enquêtés \\
\hline Guerres & 20 \\
\hline $\begin{array}{c}\text { Enrôlement dans } \\
\text { les milices }\end{array}$ & 5 \\
\hline Persécutions politiques & 5 \\
\hline Misère & 10 \\
\hline Total & $\mathbf{5 0}$ \\
\hline
\end{tabular}




\section{Des témoignages de bénévoles recueillis à Calais}

Les témoignages de bénévoles recueillis au cours des observations in situ que nous avons menées à Calais pendant une semaine en 2012, nous ont permis de mieux comprendre les mécanismes de l'enfermement dans la prison ouverte où se trouvent ces migrants.

Ces bénévoles, qui ont requis l’anonymat, ont insisté sur deux points principaux, à savoir : les conséquences de la fermeture de Sangatte et du démantèlement de la "jungle", d'une part, et les difficultés auxquelles ils sont confrontés au quotidien, d'autre part.

"La fermeture de Sangatte en 2003 et la destruction de la 'jungle' n'ont résolu aucun problème. L'ancien gouvernement, en faisant ces opérations médiatiques, n'avait qu'un objectif: rendre invisibles ces migrants qui ont trouvé d'autres squatts à Calais et dans les autres villes. Avec ces pratiques, nous assistons à l'aggravation de la situation des migrants" (N.T., association Salam).

Pour les deux bénévoles du Secours catholique que nous avons rencontrés, les gouvernements successifs doivent prendre le mal à la racine. "Pourquoi retenir à tout prix des migrants dans ces conditions épouvantables? L'urgence est de lutter, certes, contre les filières clandestines des passeurs, mais aussi contre les persécutions et les pressions que peuvent subir ces migrants dans leurs pays d'origine" (S.B., Secours catholique).

"La France devra discuter avec le Royaume-Uni pour étudier le sort de ces migrants qui sont en France malgré eux. C'est toutes les difficultés auxquelles l'Europe forteresse est confrontée, à savoir la gestion des flux de migrants aux frontières." (M.H., Secours catholique). Ces témoignages traduisent bien les réelles difficultés des bénévoles confrontés au quotidien à la gestion des flux de migrants à Calais.

\section{Conclusion}

À travers cette étude sur les migrants de Calais, c'est toute la problématique des frontières qui est revisitée. Alors qu'elle permet de délimiter géographiquement un pays par rapport à un autre, la situation de ces migrants montre que la frontière est devenue un lieu de contrôle, de surveillance et de gestion inhumain. Aujourd'hui, qui dit "frontière" dit désormais "surveillance", "contrôle", "violence", alors qu'une frontière est censée être un lieu de passage, de circulation et non d'enfermement ou de confinement. Avec la mondialisation, nous sommes entrés dans une nouvelle ère, celle des frontières de "la pénalisation des migrations", alors qu'il n'y a rien de dramatique à circuler, se déplacer d'un point $\mathrm{A}$ à un point B. Les frontières, comme le souligne Michel Agier, sont piégées par des questions identitaires qui traduisent le paradoxe de ce monde contemporain marqué par la mondialisation, la globalisation, que Marshall McLuhan qualifia de "village planétaire". Ces migrants s'inscrivent dans cette logique du village planétaire qui restaure la frontière comme un lieu de vie, de circulation et d'altérité. Une frontière est par essence ouverte au monde dans ce contexte de mondialisation où vivre en vase clos est un "danger permanent” pour les États. Elle devrait permettre un transit fluide des personnes et des biens. À la frontière, on ne s'arrête pas, on passe et, par conséquent, on devrait laisser passer sans enfermer. Les frontières de la mondialisation semblent ne pas reconnaitre aux humains le droit à la mobilité. Avec ces frontières, bouger n'est pas permis, tandis que les marchandises circulent facilement dans le cadre du libre-échange. C'est en fait une négation d'un droit fondamental qu'est celui d'aller et de venir sans être inquiété. Un paradoxe face à l'idée de la mondialisation qui prône le rapprochement des peuples et, par conséquent, leur circulation. Actuellement, la police procède à la destruction des squatts dans la ville de Calais où les migrants tentent de se réfugier. 